\title{
The effect of fiber surface treatments on the tensile and water sorption properties of polypropylene-luffa fiber composites
}

\author{
H. Demir ${ }^{\mathrm{a}}$, U. Atikler ${ }^{\mathrm{a}}$, D. Balköse ${ }^{\mathrm{a}}, \mathrm{F}$. Tıhmınlığ̆lu ${ }^{\mathrm{a}, \mathrm{b}, *}$ \\ ${ }^{a}$ Department of Chemical Engineering, Faculty of Engineering, Izmir Institute of Technology, Gülbahce Campus, 35430 Urla Izmir, Turkey \\ ${ }^{\mathrm{b}}$ Izmir Institute of Technology, Center for Materials Research, Gülbahce Campus, 35430 Urla Izmir, Turkey
}

Received 31 August 2004; revised 13 May 2005; accepted 13 May 2005

\begin{abstract}
The effects of coupling agents on the mechanical, morphological, and water sorption properties of luffa fiber (LF)/polypropylene(PP) composites were studied. In order to enhance the interfacial interactions between the PP matrix and the luffa fiber, three different types of coupling agents, (3-aminopropyl)-triethoxysilane (AS), 3-(trimethoxysilyl)-1-propanethiol (MS), and maleic anhydride grafted polypropylene (MAPP) were used. The PP composites containing 2-15 wt\% of LF were prepared in a torque rheometer. The tensile properties of the untreated and treated composites were determined as a function of filler loading. Tensile strength and Young's modulus increased with employment of the coupling agents accompanied by a decrease in water absorption with treatment due to the better adhesion between the fiber and the matrix. The maximum improvement in the mechanical properties was obtained for the MS treated LF composites. The interfacial interactions improved the filler compatibility, mechanical properties, and water resistance of composites. The improvement in the interfacial interaction was also confirmed by the Pukanszky model. Good agreement was obtained between experimental data and the model prediction. Morphological studies demonstrated that better adhesion between the fiber and the matrix was achieved especially for the MS and AS treated LF composites. Atomic force microscope (AFM) studies also showed that the surface roughness of LFs decreased with the employment of silane-coupling agents.
\end{abstract}

(C) 2005 Elsevier Ltd. All rights reserved.

Keywords: A. Polymer-matrix composites (PMCs); Fibers; B. Mechanical properties; Water absorption; D. Electron microscopy; E. Surface treatments

\section{Introduction}

In recent years, significant effort has been done to investigate the use of natural fibers as reinforcement in thermoplastic composites. Natural fiber-reinforced composites have many advantages such as light weight, reasonable strength and stiffness, renewable, and biodegradable. The composites therefore provide economical and ecological properties [1-3]. Polymer-cellulosic fiber composites are used primarily in building products such as decking, fencing, siding, and decorative trim. Another applications include infrastructure such as broadwalks, marinas, and guardrails, transportation, i.e. interior automative panels,

\footnotetext{
* Corresponding author. Tel.: +90 232750 6277; fax: +90232750 6196.

E-mail address: fundatihminlioglu@iyte.edu.tr (F. Tıhmınlığlu).
}

1359-835X/\$ - see front matter (C) 2005 Elsevier Ltd. All rights reserved. doi:10.1016/j.compositesa.2005.05.036 truck floors and head liners, and industrial and consumer applications such as pallets, play ground equipment and benches. Natural fiber-reinforced (NFR) composites are likely to be environmentally superior to glass fiberreinforced (GFR) composites in most applications also for the following reasons: (1) natural fiber production results in lower environmental impacts compared to glass fiber. The production of natural fiber-reinforced transport pallets uses $45 \%$ less energy, and results in lower emission of toxic gases $\left(\mathrm{CO}_{2}\right.$, methane, $\mathrm{SO}_{2}$, and $\left.\mathrm{CO}\right)$ than production of GFR transport pallets; (2) NFR composites have higher fiber content for equivalent performance, which reduces amount of more polluting base polymers [3].

Despite the advantages of cellulosic fibers in thermoplastics, the preparation of polymer-cellulose composite materials is handicapped by the highly hydrophilic character of these fibers, which is associated with a low compatibility of hydrophobic polymers like polypropylene, as well as with a loss of mechanical properties after moisture uptake [4-6]. Due to the poor compatibility, surface of fibers must be 
treated with coupling or compatibilizing agents to improve the interface between the fiber and the matrix. However, the use of both matrix resin (maleated polyolefins) and fiber surface treatment (coupling agents) have received considerable attention due to their effectiveness in modifying the interface by forming a link between the components $[7,8]$. Colom et al. [9] modified surface of aspen wood fibers with $\gamma$-methacryloxypropyltrimethoxy silane for the enhancement of interface of HDPE/lignocellulosic fiber. They reported that tensile strength of modified composites, having $20 \%$ lignocellulosic fiber, is $37.5 \%$ higher than that of untreated composites. Joseph et al. [10] reported that maleic anhydride treatment of PP improves tensile strength of sisal/PP composites for $15 \%$, compared to the untreated sisal/PP composites, having $20 \%$ sisal fiber. Ichazo et al. [11] also reached similar result for wood flour-PP composites. In that study, the polypropylene homopolymer matrix was modified by reactive extrusion with maleic anhydride and wood flour was modified with silanecoupling agents. Composites, modified with maleic anhydride, showed significant tensile strength, modulus and izod impact. Tensile strength of maleated polypropylene composites were $5.5-16 \%$ higher than the untreated composites. Recent researches employed different coupling agents for the modification of fiber surface such as $\gamma$-methacryloxypropyltrimethoxy silane, vinyltris(2-methoxyethoxy) silane. These coupling agents also improved the compatibility of the fiber with the polymeric matrix used [9,11-13].

This paper presents the preparation and characterization of luffa cylindrica (sponge gourd) fiber-reinforced polypropylene (PP) composites. In this study, luffa cylindrica, having interior tough fiber, was used as a natural fiber and PP was used as the thermoplastic polymeric matrix. Luffa cylindrica is a subtropical plant abundant in Asia, Central, and South America. The fruit of luffa fiber has a fibrous and vascular system that forms a natural mat when dried [14]. These fibers are readily available in the cosmetic and bath section of department stores, discount stores, pharmacies, and specialty shops. A study on the structure and morphology of the dried sponge product was reported elsewhere [14]. The recent study related to the use of luffa fiber in polyester (thermoset resin) composites was reported by Boynard et al. [15]. They investigated the effect of treatment of luffa fibers on the flexural properties of the polyester thermoset composites. To our knowledge, previously no one has reported the use of luffa fibers in thermoplastic composites as reinforcement/filler.

In this work, the effects of surface modification and luffa fiber concentration on the mechanical, morphological, and water uptake properties of polypropylene (PP) composites were investigated and reported. In order to improve the interaction between the matrix and the fibers, silanecoupling agents, namely, aminopropyltriethoxy silane and mercapto silane were employed in the pretreatment of luffa cylindrica fiber. Maleated PP was used for the improvement of surface of the PP matrix.

\section{Theory}

\subsection{Evaluation of interfacial interaction}

Interfacial interaction between the polymer matrix and the filler is an important factor affecting the mechanical properties of the composites. Thus, theoretical yield strength and ultimate tensile strength of the composites are modeled to show the effect of interfacial interaction on the tensile strength of the composites.

The effects of composition and the interfacial interaction on tensile yield stress or tensile strength of particulate filled polymers, which is described by the Pukanszky model, is indicated in Eq. (1). The parameter $B_{\sigma y}$ is an interaction parameter that is related to the macroscopic characteristics of the filler-matrix interface and interphase [16]

$\sigma_{y c} / \sigma_{y m}=\frac{1-\Phi_{\mathrm{f}}}{1+2.5 \Phi_{\mathrm{f}}} \exp \left(B_{\sigma y} \Phi_{\mathrm{f}}\right)$

where $\Phi_{\mathrm{f}}$ is the volume fraction of the filler, $\sigma_{y c}$ and $\sigma_{y m}$ denote the tensile yield stress of composite and matrix, respectively. The first term in Eq. (1) is related to the decrease in effective load bearing cross-section, while the second one is concerned with the interfacial interaction between filler and matrix. Interfacial interaction depends on the area of the interphase, and the strength of the interaction as shown in Eq. (2)

$B_{\sigma}=\left(1+A_{\mathrm{f}} \rho_{\mathrm{f}} t\right) \ln \left(\sigma_{y i} / \sigma_{y m}\right)$

where $A_{\mathrm{f}}$ is the specific surface area of the filler, $\rho_{\mathrm{f}}$ is its density, and $t$ is the thickness of the interface. From the $B_{\sigma}$ values, strength of interaction $\sigma_{y i}$ can be calculated.

\section{Experimental}

\subsection{Materials}

Isotactic PP, (MH-418, PETKIM), in the pellet form with a density of $895 \mathrm{~kg} / \mathrm{m}^{3}$, and luffa cylindrica fibers (fiber length $3-5 \mathrm{~mm}$ ) were used for the preparation of composites. Fibers were modified using two different types of coupling agents to improve compatibility of filler with polymer. The silane-coupling agents are: 3-(trimethoxysilyl)-1-propanethiol (MS), and (3-aminopropyl)-triethoxysilane (AS). Maleic anhydride grafted polypropylene (MAPP) (AdmerQF-300E) was used for the improvement of polypropylene surface. The chemical structure and supplier of these agents were given in detail in Table 1. 
Table 1

Chemical structure of the surface modifiers

\begin{tabular}{lll}
\hline Ingredients & Chemical formula & Producers \\
\hline $\begin{array}{l}\text { (3-Aminopropyl)- } \\
\text { triethoxysilane (AS) }\end{array}$ & $\mathrm{C}_{9} \mathrm{H}_{23} \mathrm{NO}_{3} \mathrm{Si}$ & Fluka Co. \\
$\begin{array}{l}\text { 3-(Trimethoxysilyl)-1- } \\
\text { propanethiol (MS) }\end{array}$ & $\mathrm{C}_{6} \mathrm{H}_{16} \mathrm{O}_{3} \mathrm{SSi}$ & Merck Co. \\
$\begin{array}{l}\text { Maleic anhydride grafted } \\
\text { PP (MAPP) }\end{array}$ & AdmerQF-300E & Kuraray Co. \\
\hline
\end{tabular}

\subsection{Pretreatment of luffa cylindrica fibers}

Luffa cylindrica fibers were obtained from local specialty shop. The LFs were washed with water to remove the adhering dirt. They were dried in an oven at $70{ }^{\circ} \mathrm{C}$ for $6 \mathrm{~h}$. After drying, they were cut with Waring Blendor for reducing the length of fiber to $2-3 \mathrm{~mm}$. Fibers were pretreated with $0.1 \mathrm{M}$ sodium hydroxide $(\mathrm{NaOH})$ solution at boiling temperature for $20 \mathrm{~min}$. Then, fibers were washed with distilled water until all sodium hydroxide was removed. After washing, they were dried in an oven at $70{ }^{\circ} \mathrm{C}$ for $6 \mathrm{~h}$.

\subsection{Surface modification of luffa fibers}

Surface modification of LF with silane-coupling agents was carried out in solution. LF was added to the solution of silane-coupling agent $(2.5 \mathrm{wt} \%)$ in $95 \mathrm{wt} \%$ ethanol and mixed for $15 \mathrm{~min}$ to let silane hydrolysis. Then, LFs were added to the mixture and mixed for $45 \mathrm{~min}$ for the condensation and chemical bonding of silanes and cellulose fibers. Treated LFs were washed with ethanol to remove the excess of coupling agents and dried in an oven at $70^{\circ} \mathrm{C}$ for $12 \mathrm{~h}$.

Surface modification of PP was conducted by using maleated PP. Maleated PP (MAPP) was mixed (2 wt $\%$ of composite) with molten PP in rheomixer during compounding at mixing temperature of $190{ }^{\circ} \mathrm{C}$, rotor speed of $60 \mathrm{rpm}$ and mixing time of $10 \mathrm{~min}$.

\subsection{Preparation of composites}

PP composites containing $2-15 \mathrm{wt} \%$ pretreated and treated LF were prepared using torque rheometer (Thermo Haake Rheomix). The composites were prepared at mixing temperature of $190{ }^{\circ} \mathrm{C}$, rotor speed of $60 \mathrm{rpm}$ and mixing time of $10 \mathrm{~min}$. During compounding, torque vs time data of the mix can be acquired through 'Convert data' software program to determine rheological response of the composites. First, PP was incorporated into the plastograph, and then previously dried fibers were introduced as soon as torque indicated melting of the polymer (about $2 \mathrm{~min}$ ). Ten minutes of mixing was enough to reach to the stabilization torque, which indicated homogeneous mixing of filler and matrix. The composition of samples used in the experiments were tabulated in Table 2. The samples taken from the torque rheometer were compression moulded using a Carver polymer press to form rectangular sheet with dimensions of $150 \times 150 \times 3 \mathrm{~mm}^{3}$. The composites were pressed gradually at $190{ }^{\circ} \mathrm{C}$ in order to avoid void and bubble formation and then pressed at 100 bar pressure at the same temperature for $10 \mathrm{~min}$. These samples were cooled to $40{ }^{\circ} \mathrm{C}$ in 6 min under the same pressure.

\subsection{Characterization}

Tensile tests of PP-LF composites were performed under ambient conditions on Testometric Universal Testing Machine with a $5 \mathrm{kN}$ load cell, and at the cross-head speed of $50 \mathrm{~mm} / \mathrm{min}$. Tensile test specimens were prepared using a dog bone shaped hollow die punch according to ASTM D-638 procedure. The test results were taken by WINTEST software program supplied from Testometric Co. At least five specimens were tested and the mean values were reported.

Scanning electron microscopy (SEM) was used to examine the morphology of the PP-LF composites. Fracture surfaces of tensile tested specimens, containing $15 \mathrm{wt} \%$ untreated and treated LF with amino silane and mercapto silane and MAPP, were analyzed with a Philips XL-305 FEG-SEM to investigate the interface between the filler and the matrix and the dispersion of filler in the matrix.

The surface topography of the untreated, sodium hydroxide and coupling agents treated LFs were investigated by using AFM Digital instrument MMSPM Nanoscope 4 . The treated and untreated LFs were compressed prior to scanning and three points for each specimen was investigated on a $10 \times 10 \mu \mathrm{m}$ surface area.

The FTIR-ATR analysis was performed by using a Digilab FTS 3000MX spectrometer with ATR attachment to analyze the interfacial reactions between fibers and silanecoupling agents. The FTIR spectra of modified luffa fibers was subtracted from the spectra of the untreated luffa fiber.

\subsection{Water uptake of the composites}

The samples were cut into $10 \times 10 \times 0.1 \mathrm{~cm}^{3}$ sheets. First, the samples were dried at $70{ }^{\circ} \mathrm{C}$ for overnight to reach the constant weight. Then, the samples were immersed into static distilled water bath at $25^{\circ} \mathrm{C}$ to observe the sorption of water. Mass uptake of the samples were measured periodically by removing them from the water bath. The water uptakes were plotted as a function of time. The samples were wiped with the tissue paper to remove the surface water before weighing. Water uptake of LFs reinforced PP composites at time $t$ was calculated using the equation below:

$\%$ Uptake $=\frac{M_{t}-M_{0}}{M_{0}} \times 100$ 


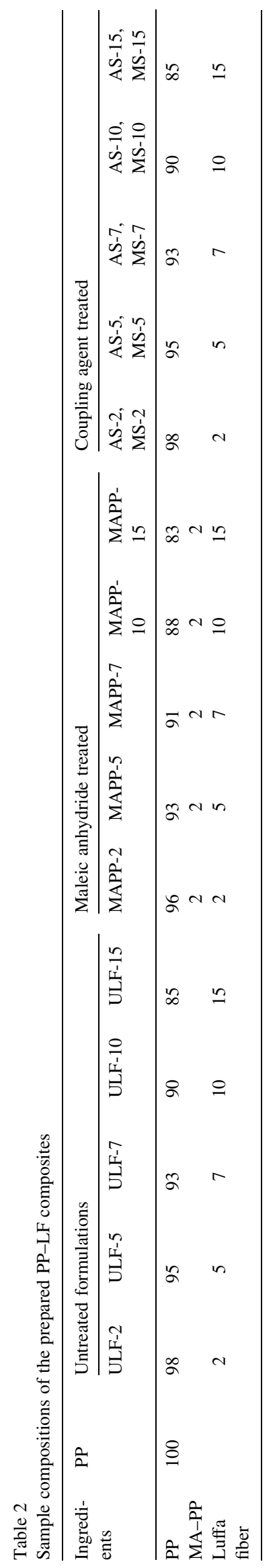

where; $M_{t}$ is mass of sample at time $t ; M_{0}$ the mass of sample at $t=0$.

\section{Results and discussion}

\subsection{Rheological properties of composites}

Since torque is an indicator of viscosity, which reveals relative rheological behavior of composites, the effect of fiber loading or surface treatment on the rheological properties of the composites can be investigated using torque vs time data at stabilization conditions. The torque vs time data for the PP-LF composites were recorded at mixing temperature of $190{ }^{\circ} \mathrm{C}$, rotor speed of $60 \mathrm{rpm}$ and mixing time of $10 \mathrm{~min}$. Fig. 1 illustrates a typical torque vs time data of the treated fiber PP composites and compares with the neat PP matrix. As seen in Fig. 1, the initial torque increased rapidly by the incorporation of polymer, which is depicted as a peak at around $40 \mathrm{~s}$. Torque decreased rapidly as soon as temperature of polypropylene increased and melting occurred. After complete melting at around $120 \mathrm{~s}$, cellulose was fed to rheomixer, which was accompanied by an increase in the viscosity. This second peak was proportional to the fiber loading. After wetting of the fibers by the polymer, the good dispersion of filler in the polymer matrix was obtained. At this point, torque values decreased up to a stable value that is called stabilization torque. Composite reached the stabilization torque at around $400 \mathrm{~s}$. A stable torque is also an indicator of homogenization of fiber in the melt [10]. When stabilization values were compared, it was clearly seen that the incorporation of fibers was accompanied by an increase in the stabilization torque for all the treatments applied. Stabilization torque was $5.62 \mathrm{~N} \mathrm{~m}$ for the neat PP whereas $6.43 \mathrm{Nm}$ for the untreated, $7.23 \mathrm{~N} \mathrm{~m}$ for MAPP, $8.73 \mathrm{~N} \mathrm{~m}$ for $\mathrm{MS}$ and $9.33 \mathrm{~N} \mathrm{~m}$ AS treated composites, respectively. The increase in torque values with surface treatment can be explained by enhanced interactions between the fiber and the polymer. Chemical bonds formed with employment of surface

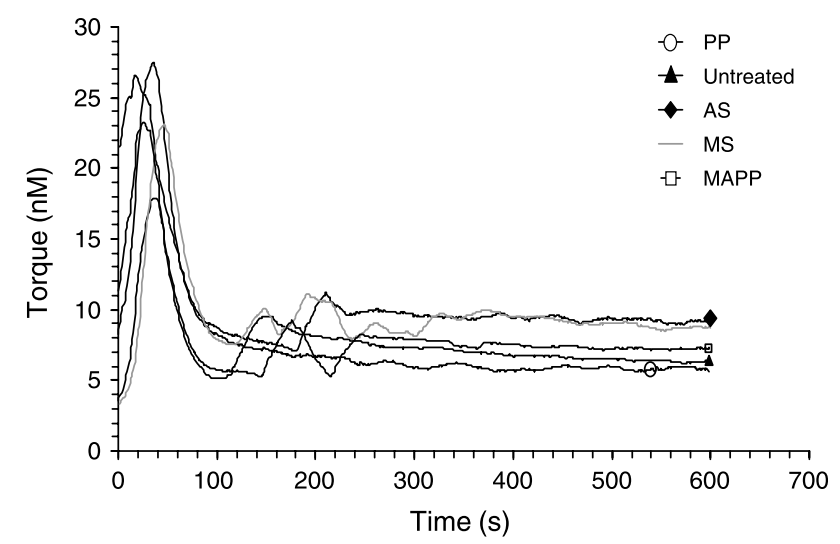

Fig. 1. Torque vs time data for pure PP and $15 \mathrm{wt} \%$ luffa fiber loaded (treated and untreated) composites. 
treatment would have increased shearing between the fiber and the matrix, hence, the stabilization torque value attained. The extend of increase was more pronounced for the MS and AS treatments compared to MAPP treatment which could be treated as an evidence for more pronounced enhancement of fiber-matrix interaction with AS and MS treatment. These two treatments also revealed better results in the mechanical response, especially in the tensile strength properties of the composites, which will be explained in Section 4.2.

\subsection{Mechanical properties}

Tensile tests were conducted to understand the effects of the fiber loading and coupling agent employment on the mechanical properties of LF-PP composites. Tensile strength, Young's modulus, and elongation at break of the PP-LF composites were measured at ambient conditions.

The importance of the treatment with coupling agent can be assessed by comparing the results of the untreated and treated composites. Fig. 2 illustrates the tensile strength of LF filled composites containing the untreated (ULF) and the treated LF's with two different silane-coupling agents, and MAPP treatment as a function of fiber loading. In general, the tensile strength of the treated and untreated composites decreased as the fiber content increased. The reduction in the tensile stress with an increase of filler content can be explained by the reduction in the effective matrix crosssection. However, it was clearly observed that the reduction in the tensile strength of PP composites has been decreased by the silane-coupling agents. The decrease is significantly greater in the untreated composites than the treated composites. The amino functional silane and mercapto silane treated fiber composites showed a reactive coupling effect that resulted in higher tensile strength compared to the untreated ones. The higher tensile strength was observed in only amino (AS) and mercapto (MS) treated LF-PP composites with $2 \mathrm{wt} \%$ fiber content. Above $2 \mathrm{wt} \%$ loading, true reinforcement cannot be attained despite the silane treatment.

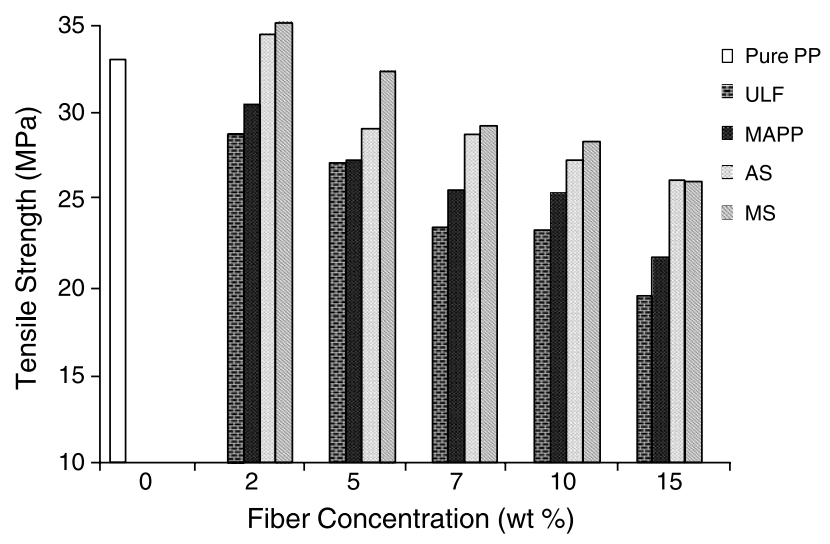

Fig. 2. Effect of surface treatment and fiber loading on the tensile strength of the composites.

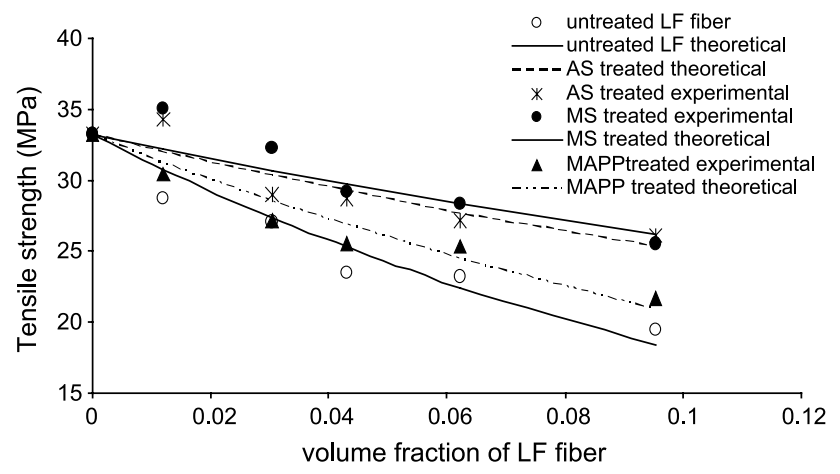

Fig. 3. Effect of surface treatment and fiber content on the experimental and theoretical tensile strength of the composites.

Tensile strength of the untreated LF-PP composites containing $15 \mathrm{wt} \%$ LF decreased from 33 to $19.5 \mathrm{MPa}$ which corresponds to the $41 \%$ decrease. For the $15 \mathrm{wt} \%$ treated fiber composites, the decrease was about $21 \%$ for the MS and the AS treated fiber composites and $11 \%$ for the MAPP treated fiber composites. Tensile strengths of the composites containing $15 \mathrm{wt} \% \mathrm{LF}$ treated with $2.5 \mathrm{wt} \%$ AS and MS increased by $33 \%$ compared to the untreated composites. For the MAPP treated composites, the enhancement is up to $11 \%$. The increase in the tensile strength with the silane treatment can be explained by the better adhesion between the filler and the matrix. Without coupling agent, the only adhesion mechanism is interdiffusion. Silane-coupling agents yields to hydrogen and covalent bonding between hydroxyl groups of filler and polysiloxanes formed by hydrogenation of silanes providing better adhesion between the fiber and the matrix. Better adhesion improves stress transfer through fibers, therefore, increases the tensile strength of composites [17].

Fig. 3 shows the comparison of experimental data of the tensile strength values of PP-LF fiber composites with the Pukanszky model. As seen in the figure, except at low volume fraction region, the model predicts the data well. Since the parameter $B$ in the model represents the strength of interaction between the PP and the LF fiber, the higher $B$ values indicates the better interaction. In literature, Pukanszky and Tudos [16] for $\mathrm{PP}-\mathrm{CaCO}_{3}$ and Metin et al. [18] for PP-zeolite composites showed that $B$ parameter can be effectively used as a quantitative measure of the efficiency of the surface treatment on the polymer matrixfiller interface. $B$ values were calculated using the experimental tensile strength data and Eq. (1). $B$ values for the untreated LF fiber and treated LF fiber with AS, MS, and MAPP were found as $-2.93,0.43,0.81$, and -1.57 , respectively. $B$ values increased with the treatment of the fiber, and the MS treated composites have the highest $B$ value indicates the strongest interaction between the polymer and the fiber compared to others.

Fig. 4 shows the Young's modulus of the composites as a function of filler content for the different treatment conditions. The Young's modulus of the composites 


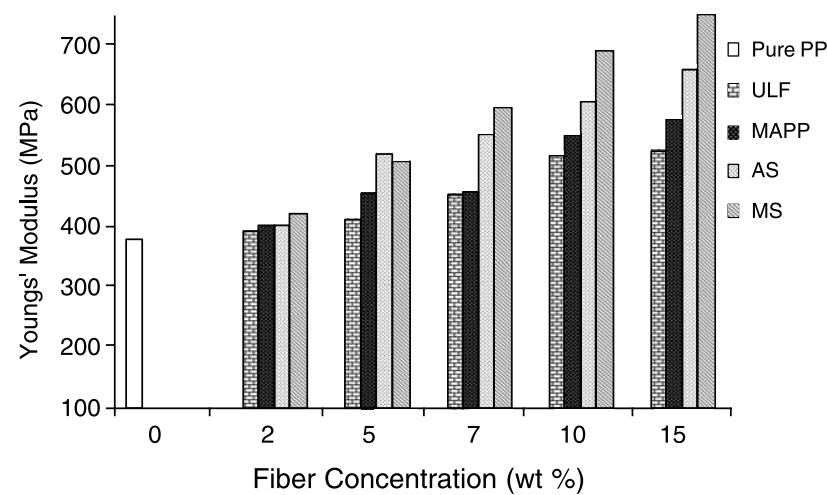

Fig. 4. Effect of surface treatment and fiber loading on the Youngs' modulus of the composites.

increased as the fiber loading increased. Young's modulus of the composites containing $15 \mathrm{wt} \%$ fiber increased by 38 , 52, 74, and $98 \%$ for the untreated, MAPP, AS, and MS treated composites, respectively. The highest increase was achieved for the MS treated composites, followed by the AS treated composites. The increase in the Young's modulus due to the silane treatment can be attributed to the better adhesion between the fiber and the matrix by chemical interactions. Better adhesion yields to more restriction to deformation capacity of the matrix in the elastic zone increasing Young's modulus.

Variation of elongation at break values as a function of filler content for different coupling agent treatment was illustrated in Fig. 5. As shown in Fig. 5, the elongation at break values for all composites decreased with the increase in LF loading. Incorporation of even low fraction ( $2 \mathrm{wt} \%)$ of fiber to the matrix caused a sharp decrease in the elongation at break values. Elongation at break of pure PP is around $338 \%$, whereas elongation at break of $2 \mathrm{wt} \%$ fiber loaded composites is around $17 \%$, almost independent of coupling agent employment. The decrease in the elongation at break was much more pronounced for AS and MS treated composites due to the adhesion between fiber and matrix restricts deformation capacity of matrix in the elastic zone as well as the plastic zone.

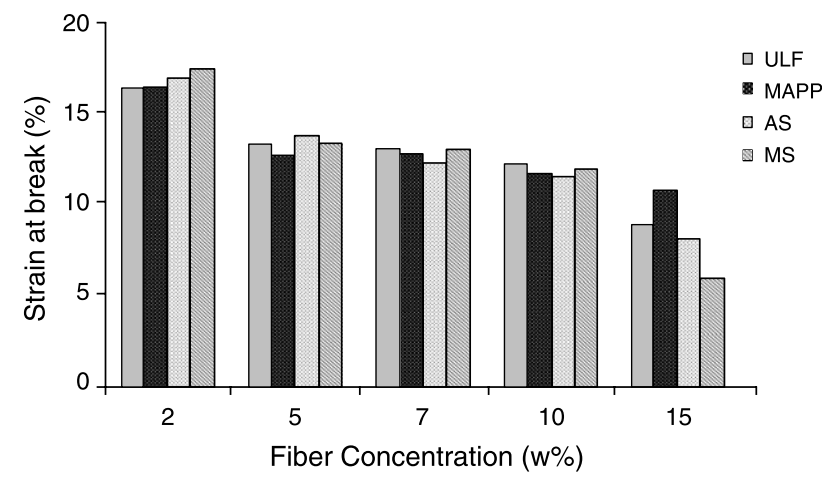

Fig. 5. Effect of surface treatment and fiber loading on elongation at break of the composites.

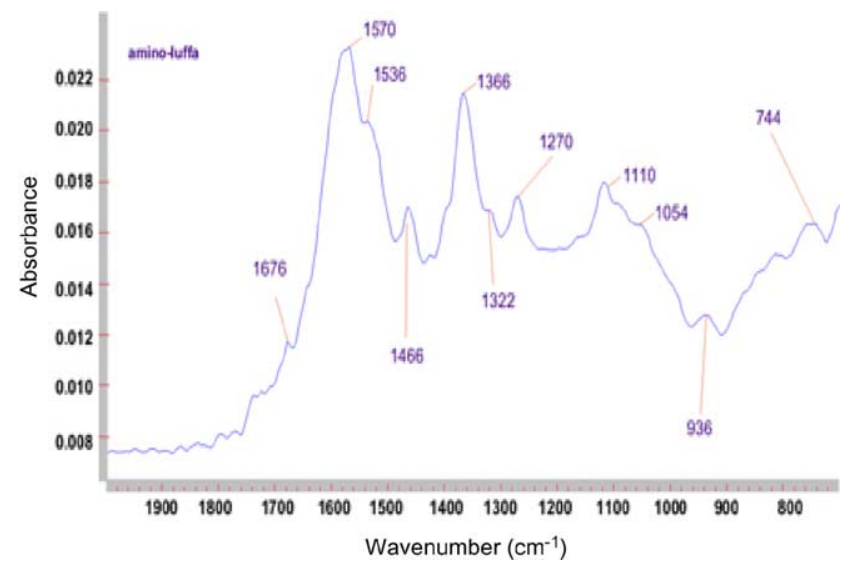

Fig. 6. FTIR-ATR spectra of amino modified luffa fiber.

\subsection{Characterization of modified fibers by FTIR}

The subtraction spectra of the AS and MS modified luffa fibers were shown in Figs. 6 and 7, respectively. The broad bands around 950 and $1150 \mathrm{~cm}^{-1}$ are attributed to asymmetric stretching of $\mathrm{Si}-\mathrm{O}-\mathrm{Si}$ linkage and $\mathrm{Si}-\mathrm{O}-$ cellulose bonds for AS modified luffa fibers shown in Fig. 6. These bands prove that condensation of silanols and chemical bonding of silane groups to cellulose was achieved via silane treatment. The absorption bands at 936 and $1366 \mathrm{~cm}^{-1}$ also confirm the presence of the $\mathrm{Si}-\mathrm{O}-$-cellulose bond. The band at $1370 \mathrm{~cm}^{-1}$ belongs to deformation of $\mathrm{NH}_{2}$ that characteristic peak of amino silane. The asymmetric stretching of $\mathrm{Si}-\mathrm{O}-\mathrm{Si}$ linkage and $\mathrm{Si}-\mathrm{O}-$ cellulose bonds were also observed around 950 and $1150 \mathrm{~cm}^{-1}$ for MS treated luffa fibers as shown in Fig. 7. The existence of 1200 and $1366 \mathrm{~cm}^{-1}$ bands could be attributed to the presence of $\mathrm{Si}-\mathrm{O}-\mathrm{Si}$ and $\mathrm{Si}-\mathrm{O}-$ cellulose bonds as similar to amino treated luffa fibers [19-21].

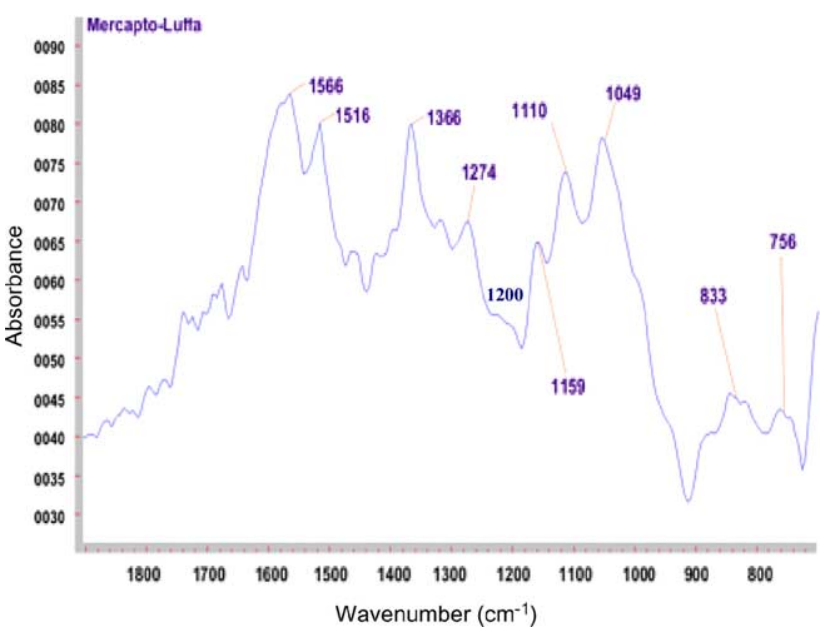

Fig. 7. FTIR-ATR spectra of mercapto modified luffa fiber. 


\subsection{Morphology}

The effect of the surface treatment on the interface between the PP and the LF's was studied by examining the fracture surfaces of the tensile tested composites with SEM. SEM micrographs of the fracture surface of the treated and untreated composites containing $15 \mathrm{wt} \% \mathrm{LF}$ can be seen in Fig. 8(a)-(h). Both treated and untreated LFs distributed in transverse and longitudinal directions in the polymeric matrix and fibers are well dispersed in the matrix indicating the efficient mixing of filler within the polymeric matrix. The micrographs illustrate the differences in microstructure
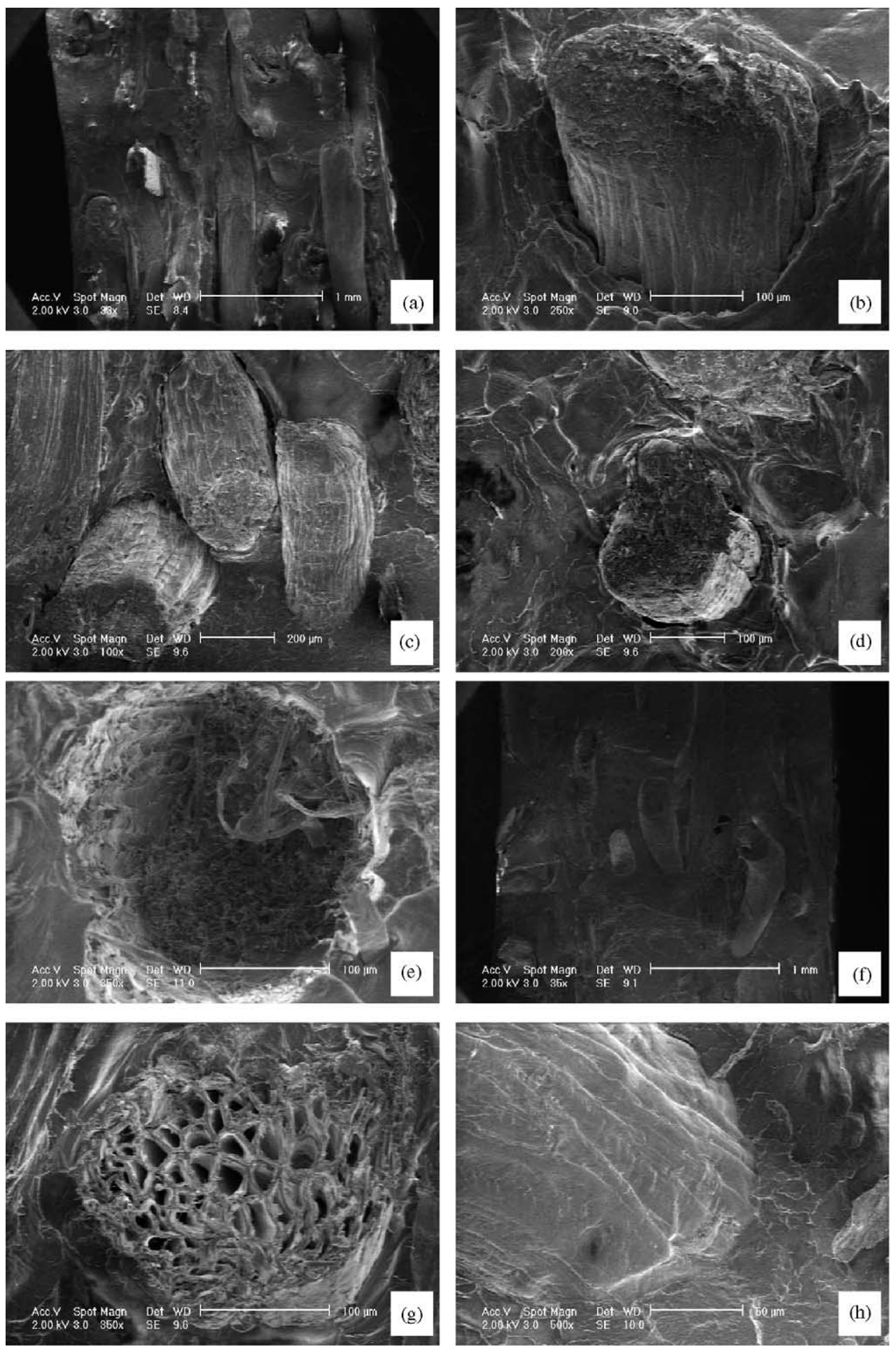

Fig. 8. SEM micrographs of the fracture surface of PP-LF composites containing 15 wt $\%$ (a and b) untreated LF; (c and d) maleated PP; (e and f) 2 wt $\%$ AS treated; (g and h) MS treated. 

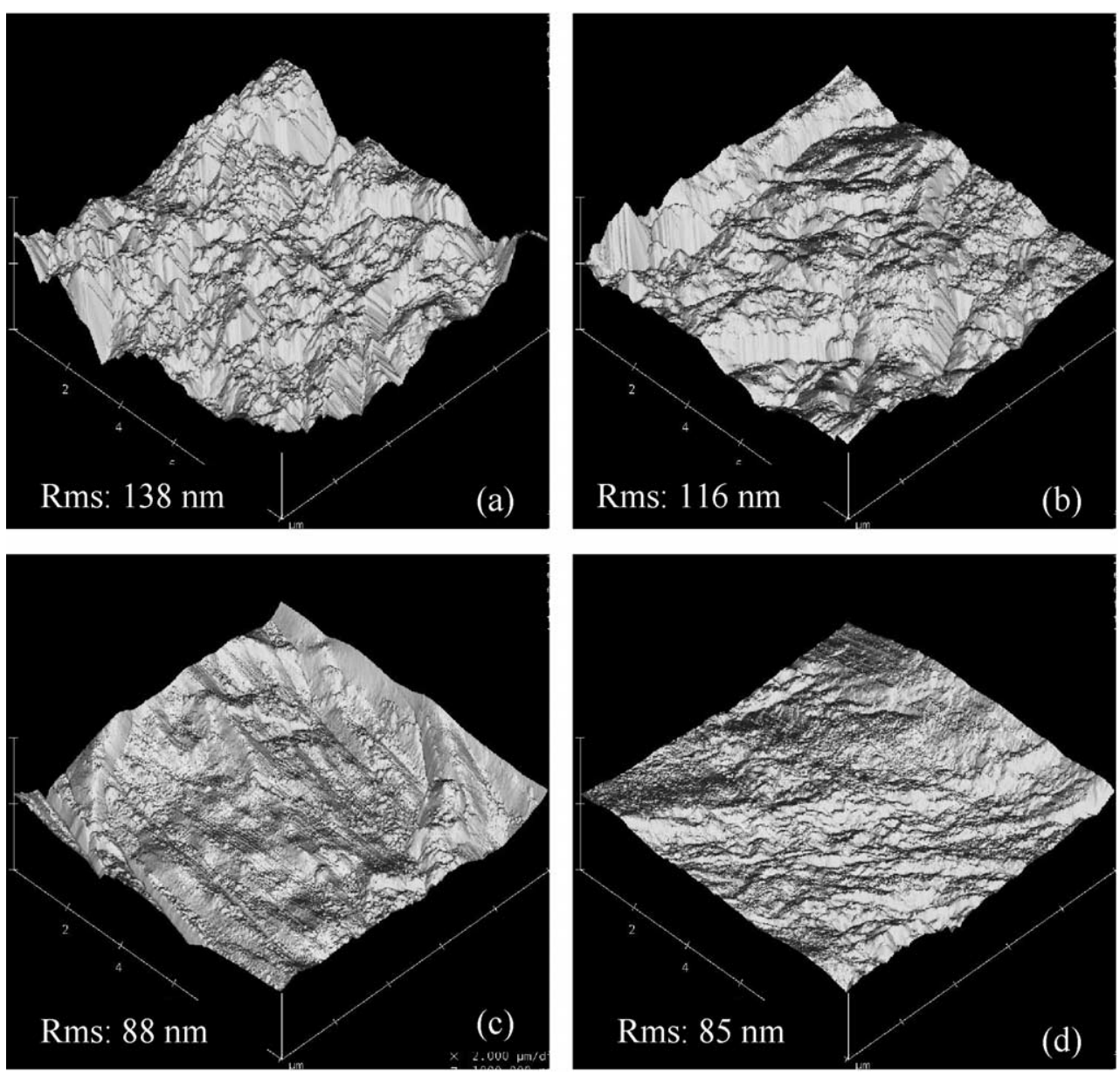

Fig. 9. Atomic force microscope (AFM) topographic pictures of (a) untreated; (b) $\mathrm{NaOH}$ treated; $(\mathrm{c}) \mathrm{NaOH}+\mathrm{AS}$ silane treated; and (d) $\mathrm{NaOH}+\mathrm{MS}$ treated LFs.

of composites. Examination of fracture surface of untreated LF composites (Fig. 8(a) and (b)) indicates that there are voids between fiber and matrix which is an evidence of poor adhesion. Poor adhesion seems to facilitate debonding of the fiber. This was also confirmed by the mode of fracture in the untreated composites. Fracture seems to be dominated by matrix failure since no fiber breakage can be observed. SEM micrographs of the treated composites clearly indicated that the treatment facilitates good adhesion between fiber and matrix. Fiber-matrix adhesion seemed to be better for MAPP treated composites than the untreated LF composites as shown in Fig. 8(c) and (d). In fact there are still voids around the fiber to a lesser extend compared to ULF composites. Fracture mechanism was still dominated by matrix failure since no fiber breakage could be observed. Fracture surface examinations of silane treated composites exhibited the best results in terms of interfacial adhesion. The AS treated and the MS treated composites are illustrated in Fig. 8(e)-(h), respectively. As shown in Fig. 8(f), AS treated fiber was well surrounded by the matrix without voids. The fracture surface of AS treated composites illustrate that failure of composites takes place by a fiber breakage. At the same time, no interfacial failure was observed. When fiber breakage occurs, matrix fibrillation takes place, which is a good indicator of better interfacial adhesion between fiber and matrix as shown in Fig. 8(e). Examination of fracture surface of MS treated composites lead to similar results with the AS treated composites with fiber failure (Fig. 8(g)) and good adhesion between fiber and matrix (Fig. 8(h)). All these observations are consistent with the mechanical test results.

AFM images of natural, sodium hydroxide treated and silane treated LFs were shown in Fig. 9. AFM pictures illustrate the reduction of roughness via surface treatment of fibers. Untreated fibers exhibit a roughness value of $138 \mathrm{~nm}$, whereas the AS and the MS treated fibers exhibit 88 and $85 \mathrm{~nm}$ of surface roughness, respectively. These results can be accepted as a proof for the surface coverage of the fibers with a siloxane layer resulting a decrease in the surface roughness. $\mathrm{NaOH}$ treatment did not cause a significant variation in roughness of LFs.

\subsection{Water sorption}

Fig. 10 shows water absorption of $10 \mathrm{wt} \%$ untreated and treated LF loaded composites as a function of time. It is 


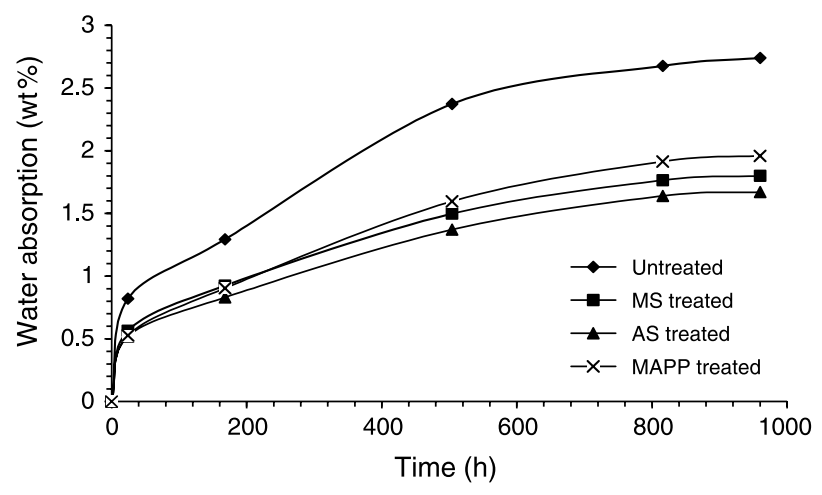

Fig. 10. Effect of treatment on the water absorption of PP/LF composites containing $10 \mathrm{wt} \% \mathrm{LF}$.

obvious that treatment of the fibers with silanes and MAPP reduced water absorption of the composites. The untreated composites exhibited $2.8 \%$ water absorption when immersed in distilled water for 40 days. The decrease in water absorption is $34.3,39.0$, and $28.4 \%$ for MS, AS, and MAPP treated composites, respectively. Water absorption in cellulose fibers is caused by hydrogen bonding between free hydroxyl groups on cellulose molecules and water molecules. Silane-coupling agents and maleic anhydride group on MAPP form hydrogen or covalent bonds with some of free hydroxyl groups of cellulose, which reduce the water absorption capacity of cellulose. Another reason for the decrease in water absorption capacity of composites would be enhanced adhesion between fiber and matrix by the treatment that results in a decrease in voids between fiber and polymer matrix. Poor adhesion causes cracks and voids between the polymeric matrix and the luffa fiber. This causes easy penetration and storage of water through the voids. The volume of voids decrease due to enhanced adhesion and therefore water penetration or storage through the interface is restricted. Silane and MAPP treated composites with lesser water absorption values have greater tensile strength, confirming better interfacial adhesion via bonding between fiber and coupling agent.

\section{Conclusions}

Effects of coupling agents on the mechanical, morphological, and water absorption properties of luffa fiber (LF) /polypropylene (PP) composites were studied to enhance the interfacial interactions between the PP matrix and the luffa fibers. Mechanical test results clearly showed that both silane treatment of LFs and reactive treatment of composite with MAPP during compounding increased the tensile strength and Young's modulus. Composites containing MS treated luffa fiber showed the most pronounced improvement in the mechanical properties compared to the composites containing untreated LF's due to adhesion and compatibility between the PP and the silane treated LF. The improvement in adhesion between PP and treated LF fiber with coupling agents was also confirmed with the semiempirical model of the Pukanzy and was is in agreement with the experimental data and was also supported by scanning electron microscopy (SEM).

AFM studies of surface of the fibers showed that silane treatment decreased surface roughness of the fibers. This is also an indication of the surface coverage of the fibers with a silane layer. Water absorption results showed that silane and MAPP treatment reduced the water absorption capacity compared to untreated composites. Water sorption results can be correlated with mechanical test results, which can be treated as a proof for enhanced interfacial interactions with employment of treatment.

\section{Acknowledgements}

The authors express their thanks to the staff of Center for Materials Research at Izmir Institute of Technology for SEM and AFM measurements.

\section{References}

[1] Van de Velde K, Kietkens P. Thermoplastic polymers: overview of several properties and their cosequences in flax fibre reinforced composites. Polym Test 2001;20:885-93.

[2] Lundquist L, Marque B, Hagstrand PO, Leterrier Y, Manson JAE. Novel pulp fibre reinforced thermoplastic composites. Compos Sci Technol 2003;63:137-52.

[3] Joshi VS, Drzal TL, Mohanty KA, Arora S. Are natural fiber composites environmentally superior to glass fiber reinforced composites? Composites Part A 2004;35:371-6.

[4] Tajvidi M, Ebrahimi G. Water uptake and mechanical characteristics and natural filler-polypropylene composites. J Appl Polym Sci 2003; 88:941-6.

[5] Bledzki KA, Reihmane S, Gassan J. Thermoplastics reinforced with wood fillers: a literature review. Polym Plast Technol 1998;37: 451-68.

[6] Khan AM, Ali IKM. Swelling and thermal conductivity of wood and wood-plastic composite. Polym Plast Technol 1997;36:179-87.

[7] Felix MJ, Gatenholm P. The nature of adhesion in composites of modified cellulose fibers and polypropylene. J Appl Polym Sci 1991; 42:609-20.

[8] Zafeiropoulos EN, Baille CA, Hodgkinson JM. Engineering and characterisation of the interface in flax fibre/polypropylene composite materials. Part II. The effect of surface treatments on the interface. Composites Part A 2002;33:1185-90.

[9] Colom X, Carrasco F, Pages P, Canavate J. Effects of different treatments on the interface of HDPE/lignocellulosic fiber composites. Compos Sci Technol 2003;63:161-9.

[10] Joseph PV, Joseph K, Thomas S. Effect of processing variables on the mechanical properties of sisal-fiber-reinforced polypropylene composites. Compos Sci Technol 1999;59:1625-40.

[11] Ichazo MN, Albano C, Gonzales J, Perera J, Candal MV. Polypropylene/wood flour composites: treatments and properties. Compos Struct 2001;54:207-14.

[12] Wu J, Yu D, Chan CM, Kim J, Ma YW. Effect of fiber pretreatment condition on the interfacial strength and mechanical properties of wood fiber/PP composites. J Appl Polym Sci 2000;76:1000-10.

[13] Coutinho MBF, Costa HST. Performance of polypropylene-wood fiber composites. Polym Test 1999;18:581-7. 
[14] Boynard CA, d'Almeida JRM. Morphological characterization and mechanical behavior of sponge gourd (luffa cylindrica)polyester composite materials. Polym Plast Technol Eng 2000; 39(3):489-99.

[15] Boynard CA, Monteiro SN, d'Almeida JRM. Aspects of alkali treatment of sponge gourd (luffa cylindrica) fibers on the flexural properties of polyester matrix composites. J Appl Polym Sci 2003;87: 1927-32.

[16] Pukanszky B, Tudos F. The possible mechanisms of polymer-filler interaction in polypropylene- $\mathrm{CaCO}_{3}$ composites. J Mater Sci Lett 1989;8:1040-2.

[17] Hull D, Clyne WT. In: An introduction to composite material. 2nd ed. Cambridge: Cambridge University Press, UK; 1996.
[18] Metin D, Tıhmınlıoğlu F, Balköse D, Ülkü S. The effect of interfacial interactions on the mechanical properties of polypropylene/natural zeolite composite. Composites Part A 2004;35:23-32.

[19] Valadez-Gonzalez A, Cervantes-Uc JM, Olayo R, Herrera-Franco PJ. Chemical modification of henequen fibers with an organosilane coupling agent. Composites Part B 1999;30:321-31.

[20] Yang R, Liu Y, Wang K, Yu J. Characterization of surface interaction of inorganic fillers with silane coupling agents. J Anal Appl Pyrol 2003;70:413-25.

[21] Abdelmouleh M, Boufi S, Belgacem MN, Duarte AP, Ben Salah A, Gandini A. Modification of cellulosic fibers with functionalised silanes: development of surface properties. Int J Adhes Adhes 2004; 24:43-54. 